\title{
Myocardial Functional Disorders in Hemodialysis Patient with Intradialytic Hypertension
}

Mohamed E. Ibrahim, EL-Metwally L. EL-Shehawy, Hassan G. Abdelsalam, Ahmed E. Mansour, Nashwa G. Mohamed

\begin{abstract}
Department of internal medicine, Benha Faculty of Medicine, Benha University, Egypt.
\end{abstract}

Correspondence to: Nashwa G. Mohamed, Department of internal medicine, Benha Faculty of Medicine, Benha University, Egypt.

\section{Email:}

nosha9690@gmail.com

Received: 23 October 2019

Accepted: 28 December 2019

\begin{abstract}
:
Background: Hemodialysis is the process of cleaning blood outside the body. It involves taking blood from a blood vessel and passing it through a synthetic filter, known as a dialyzer. Intradialytic hypertension (IDH) is a phenomenon where blood pressure increases during hemodialysis, and is associated with increased mortality. Majority of patients with IDH suffer and die from cardiovascular complications. Aim of the work: The aim of the work is to estimate the incidence of IDH among the hemodialysis patients, and to study myocardial functional disorders during hemodialysis in IDH patients. Patient and Methods: study was carried out in hemodialysis unit, internal medicine department of Benha University Hospital, where 100 patients were selected, conventional echocardiography was done immediately before and after HD, Results: incidence of IDH was 30\%. Mean LVEDD significantly decreased post dialysis from $48.3 \mathrm{~mm}$ to $46.6 \mathrm{~mm}$. $\mathrm{P}$ value was <0.001. Mean LVESD significantly decreased from $39 \mathrm{~mm}$ to $37.1 \mathrm{~mm}$. P value was $<0.001$ Mean LAV significantly decreased from $50.6 \mathrm{~mm} 3$ to 40.8 mm3. P value was <0.001 Mean COP significantly decreased from $5.9 \mathrm{~L} / \mathrm{m}$ to $5.6 \mathrm{~L} / \mathrm{m}$. P value was <0.001 Mean SV
\end{abstract} significantly decreased from $82.5 \mathrm{ml}$ to $69.5 \mathrm{ml}$. P value was <0.001 Mean LA diameter significantly decreased from $38.2 \mathrm{~mm}$ to $32.5 \mathrm{~mm}$. $\mathrm{P}$ value was $<0.001$ in IDH patient Conclusion: IDH is a common alteration in the dialysis population with significant changes on ECHO parameter.

Keywords: Hemodialysis, IDH, LVH, echocardiographic changes 


\section{Abbreviation :}

CKD: Chronic kidney disease

HD: hemodialysis

IDH: intradialytic HTN

HTN: hypertention

LVH: Left Ventricular Hypertrophy

GFR: glomerular filtration rate

\section{Introduction}

Hemodialysis is the choice of renal replacement therapy for patients who need dialysis acutely and for many patients as maintenance therapy. It provides excellent, rapid clearance of solutes (1)

The adverse outcomes associated with large decreases in BP during hemodialysis are well known, but nephrologists should be aware of the clinical significance of increases in BP during hemodialysis (2). Intradialytic hypertension is an increase in $\mathrm{BP}$ from pre to post-hemodialysis that has been shown to be associated with poor outcomes (3).

Numerous studies have explored the possible mechanisms responsible for intradialytic hypertension yielding potential interventions to consider improving outcomes in this high-risk group of patients (4), but very little data exist on the complex phenomenon of intradialytic hypertension although this complication has been recognized for many years (3).
Factors that might be involved in the pathogenesis of intradialytic hypertension include the following: extra cellular fluid volume overload, increased cardiac output, activation of the renin-angiotensinaldosterone system (RAAS), activation of the sympathetic nervous system, endothelial cell dysfunction, peripheral vasoconstriction, fluctuations in electrolyte levels during dialysis, removal of antihypertensive medications by hemodialysis, and use of erythropoiesisstimulating agents (5).

Cardiovascular diseases are common in patients with (ESRD) and are the main causes of morbidity and mortality. A large body of evidence has occrued indicating that a portion of this cardiovascular damage may be due to hemodialysis (6).

\section{Patients and Methods}

This comparative study was carried out in hemodialysis unit, internal medicine department of Benha University Hospital, between May 2018 to May 2019, on 100 selected patients.

Approval of ethical committee was obtained and all of patients were subjected to the following procedures after taking their consents. 


\section{I-History taking included:}

Sex, Age (years), Age of starting of hemodialysis (years), UF volume and Blood flow rate.

\section{II-Clinical examination:}

1-Blood pressure measurement:-

Blood pressure was measured by sphygmomanometer. Measurements were obtained before hemodialysis, every 30 minutes during hemodialysis, and after hemodialysis.

For each hemodialysis treatment, we calculated the change in systolic blood pressure from pre- to post-dialysis. Intradialytic hypertension is defined as an increase in systolic blood pressure $\geq 10$ $\mathrm{mmHg}$ from pre- to post-dialysis in an individual treatment. Individual patients who had a change in systolic blood pressure from pre- to post-dialysis $\geq+10$ $\mathrm{mmHg}$ when averaged throughout the entire study period were defined as having persistent intradialytic hypertension.

2- Pulse rate (beat per min)

\section{III-Laboratory investigation:}

1- Biochemical tests:

Serum albumin, serum creatinine, serum K, serum $\mathrm{Na}, \mathrm{HCO} 3, \mathrm{PTH}$, Ferritin, $\mathrm{Ca}, \mathrm{P}$, WBC. Hemoglobin, Hct (\%),CRP and Mg . 2- Imaging
ECG and Echocardiographic Assessment:

Conventional echocardiography was done immediately before and after HD with the use of a PHILIPS EPIC7 (Philips healthcare, Sanjon, Canada) echocardiography machine with a transducer (3.5 MHz).

All echocardiographic data were calculated based on the American Society of Echocardiography Guidelines

\section{Statistical methods}

Data management and statistical analysis were done using SPSS vs.25. (IBM, Armonk, New York, USA).

Numerical data were summarized as mean and standard deviation or median and range. Categorical data were summarized as numbers and percentages.

Comparisons between SBP and DBP at different times were done using repeated measure ANOVA. Different parameters were compared pre and post dialysis using paired t test.

Correlation analysis was done using Spearman's correlation. " $r$ " is the correlation coefficient. It ranges from -1 to +1 . -1 indicates perfect negative correlation, +1 indicates perfect positive correlation and 0 indicates no correlation. All $\mathrm{P}$ values were two sided. $\mathrm{P}$ values less than 0.05 were considered significant. 


\section{Results}

$>\quad$ Mean age of the whole study group was $(46 \pm 14)$ years old, $52 \%$ of study population were males while $48 \%$ were females.

$>\quad$ Diastolic blood pressure increased in $31 \%$ of patients $\{(5)-(20)\}$ and decreased in $26 \%$ of patients $\{(-5)-(-$ 15)\}. No changes were recorded in $43 \%$ of patients. (Table 1)

$>$ In those with increased SBP post dialysis, mean SBP was 128 pre dialysis and 138 at 120 minutes and 143 postdialysis. Median percent change was $13.2 \%$ and ranged from $6.7 \%$ to $27.3 \%$. In those with decreased SBP postdialysis, mean SBP was $132 \mathrm{~mm} \mathrm{Hg}$ pre dialysis and $121 \mathrm{~mm} \mathrm{Hg}$ at 120 minutes and $109 \mathrm{~mm} \mathrm{Hg}$ post dialysis. Median percent change was $-15.4 \%$ and ranged from $-23.1 \%$ to $-8.3 \%$. In those with no change in systolic blood pressure, mean SBP was $129 \mathrm{mmHg}$ pre dialysis at 120 minutes and post dialysis. (Table 2)

Mean serum $\mathrm{Ca}++$ level was $7.67 \pm$ $0.3 \mathrm{mg} / \mathrm{dl}$ in intradialytic HTN patient, and $7.40 \pm 0.58 \mathrm{mg} / \mathrm{dl}$ in non-intradialytic HTN patient. There was no significant differences in serum $\mathrm{Ca}++$ level between intradialytic HTN patient and nonintradialytic HTN patient ( $p$ value was
0.936). Mean serum $P$ level was $5.3 \pm 0.75$ $\mathrm{mg} / \mathrm{dL}$ in intradialytic HTN patient, and $5.31 \pm 0.70 \mathrm{mg} / \mathrm{dL}$ in non-intradialytic HTN patient. There was no significant differences in serum $\mathrm{P}$ level between intradialytic HTN patient and nonintradialytic HTN patient ( $p$ value was 0.676). Mean serum PTH level was $381.6 \pm 127.47 \mathrm{pg} / \mathrm{mL}$ in intradialytic HTN patient. Mean serum PTH level was $355.26 \pm 117.15 \mathrm{pg} / \mathrm{mL}$ in non-intradialytic HTN patient. There was no significant differences in serum PTH level between intradialytic HTN patient and nonintradialytic HTN patient (p value was 0.433). Mean serum $\mathrm{Mg}$ level was $2.26 \pm 0.16 \mathrm{mg} / \mathrm{dL}$ in intradialytic HTN patient. and $2.256 \pm 0.16 \mathrm{mg} / \mathrm{dL}$ in nonintradialytic HTN. There was no significant differences in serum $\mathrm{Mg}$ level between intradialytic HTN patient and non-intradialytic HTN patient ( $\mathrm{p}$ value was 0.530$)$. No significant differences in serum albumin, serum creatinine, serum $\mathrm{K}+$, serum $\mathrm{Na}+$, serum $\mathrm{HCO} 3$, serum ferritin, O2 saturation, WBCs count, hemoglobin level, hematocrit level, serum CRP level between intradialytic HTN patient and non-intradialytic HTN patient.

\section{(Table 3)}


Twenty eight 28 percentage of patients showed LVH, $20 \%$ showed Rt $\mathrm{VH}, 16 \%$ showed inverted $\mathrm{T}$ wave and pathological Q while $12 \%$ showed inverted $\mathrm{T}$ wave only. (Table 4)

Mean LVEDD significantly decreased post dialysis from $48.3 \mathrm{~mm}$ to $46.6 \mathrm{~mm}$, (p value was <0.001). Mean LVESD significantly decreased from 39 $\mathrm{mm}$ to $37.1 \mathrm{~mm}$ (p value was <0.001). Mean LAV significantly decreased from $50.6 \mathrm{~mm}^{3}$ to $40.8 \mathrm{~mm}^{3}$. ( $\mathrm{p}$ value was $<0.001)$. Mean COP significantly decreased from $5.9 \mathrm{~L} / \mathrm{m}$ to $5.6 \mathrm{~L} / \mathrm{m}$. (p value was $<0.001)$. Mean SV significantly decreased from $82.5 \mathrm{ml}$ to $69.5 \mathrm{ml}$ (p value was <0.00). Mean LA diameter significantly decreased from $38.2 \mathrm{~mm}$ to $32.5 \mathrm{~mm}$ (p value was $<0.001$ ) in patients with intradialytic HTN. (Table 5)

$>$ Mean LVEDD significantly decreased post dialysis in intradialytic HTN patient and non-intradialytic HTN patient ( $\mathrm{p}$ value 0.046). Mean LAV significantly decreased post dialysis in intradialytic HTN patient and nonintradialytic HTN patient (p value 0.026).

\section{(Table 6)}

Table (1): Diastolic blood pressure status post dialysis

\begin{tabular}{llc}
\hline & & $\mathbf{n}(\%)$ \\
\hline DBP status & Increased $\{(\mathbf{5})-(\mathbf{2 0}) \mathbf{m m H g}\}$ & $31(31.0)$ \\
& Decreased $\{(-\mathbf{5})-(-\mathbf{1 5}) \mathbf{m m H g}\}$ & $26(26.0)$ \\
& No change & $43(43.0)$ \\
\hline
\end{tabular}

Table (2): SBP in pre dialysis and post dialysis patient

\begin{tabular}{|c|c|c|}
\hline & & SBP (mmHg) \\
\hline \multicolumn{3}{|l|}{ I. In those with increased SBP } \\
\hline Pre $(110-150 \mathrm{mmHg})$ & Mean \pm SD & $128 \pm 10$ \\
\hline 120 minutes $(120-160 \mathrm{mmHg})$ & Mean \pm SD & $138 \pm 10$ \\
\hline Post (130-160 mmHg) & Mean \pm SD & $143 \pm 8$ \\
\hline$\%$ change & Median (range) & $13.2(6.7-27.3)$ \\
\hline \multicolumn{3}{|l|}{ II. In those with decreased SBP } \\
\hline Pre $(120-160 \mathrm{mmHg})$ & Mean \pm SD & $132 \pm 9$ \\
\hline 120 minutes $(110-150 \mathrm{mmHg})$ & Mean \pm SD & $121 \pm 9$ \\
\hline Post $(100-140 \mathrm{mmHg})$ & Mean \pm SD & $109 \pm 8$ \\
\hline$\%$ change & Median (range) & $-15.4(-23.1--8.3)$ \\
\hline \multicolumn{3}{|l|}{ III. In those with ISO SBP } \\
\hline Pre $(120-140 \mathrm{mmHg})$ & Mean \pm SD & $129 \pm 7$ \\
\hline 120 minutes $(120-140 \mathrm{mmHg})$ & Mean \pm SD & $129 \pm 7$ \\
\hline Post (120-140 mmHg) & Mean \pm SD & $129 \pm 7$ \\
\hline$\%$ change & Median (range) & $0\left(\begin{array}{ll}0 & -0\end{array}\right)$ \\
\hline
\end{tabular}


Benha medical journal,vol.37, Special issue (Internal medicine and Hepatology),2020

Table (3): Laboratory findings in patient with intradialytic HTN and non-intradialytic HTN

\begin{tabular}{|c|c|c|c|c|}
\hline Laboratory findings & $\begin{array}{c}\text { Mean and } \\
\text { Standard Deviation }\end{array}$ & $\begin{array}{c}\text { patient with } \\
\text { intradialytic HTN }\end{array}$ & $\begin{array}{c}\text { Patient non } \\
\text { intradialytic HTN }\end{array}$ & $P$ value \\
\hline Serum albumin $(\mathrm{gm} / \mathrm{dl})$ & Mean \pm SD & $3 \pm 0.8$ & $2.974 \pm 0.62$ & 0.467 \\
\hline Serum creatinine $(\mathrm{mg} / \mathrm{dL})$ & Mean \pm SD & $8 \pm 3.25$ & $9.30 \pm 2.92$ & 0.808 \\
\hline Serum $\mathbf{K}^{+}(\mathrm{mEq} / \mathrm{L})$ & Mean \pm SD & $5.2 \pm 0.544$ & $5.119 \pm 0.73$ & 0.248 \\
\hline Serum $\mathrm{Na}^{+}(\mathrm{mEq} / \mathrm{L})$ & Mean \pm SD & $136.13 \pm 2.52$ & $136.62 \pm 2.73$ & 0.519 \\
\hline $\mathbf{H C O}_{3}(\mathrm{mEq} / \mathrm{L})$ & Mean \pm SD & $17.8 \pm 1.71$ & $17.40 \pm 1.39$ & 0.672 \\
\hline Serum $\mathbf{C a}^{++}(\mathrm{mg} / \mathrm{dl})$ & Mean \pm SD & $7.67 \pm 0.3$ & $7.40 \pm 0.58$ & 0.936 \\
\hline Serum $\mathbf{P}^{+}(\mathrm{mg} / \mathrm{dL})$ & Mean \pm SD & $5.3 \pm 0.75$ & $5.31 \pm 0.70$ & 0.676 \\
\hline Serum PTH ( pg/mL) & Mean \pm SD & $381.6 \pm 127.47$ & $355.26 \pm 117.15$ & 0.433 \\
\hline Serum Ferritin( ng/mL) & Mean \pm SD & $124.93 \pm 45.47$ & $113.06 \pm 35.03$ & 0.904 \\
\hline $\mathbf{O}_{2}$ saturation $(\%)$ & Mean \pm SD & $92.70 \pm 1.643$ & $92.96 \pm 1.38$ & 0.740 \\
\hline Serum $\mathbf{W B C}_{\mathrm{s}}(\mathrm{m} / \mathrm{mm} 3)$ & Mean \pm SD & $7646.67 \pm 3958.9$ & $8031.43 \pm 4060.97$ & 0.222 \\
\hline Serum Hemoglobin( $\mathrm{g} / \mathrm{dl})$ & Mean \pm SD & $8.637 \pm 0.694$ & $8.493 \pm 0.69$ & 0.367 \\
\hline Serum Hematocrit (\%) & Mean \pm SD & $23.30 \pm 2.12$ & $23.069 \pm 1.69$ & 0.891 \\
\hline Serum CRP( mg/l) & Mean \pm SD & $8.80 \pm 8.31$ & $9.34 \pm 8.43$ & 0.384 \\
\hline Serum Mg( mg/dL) & Mean \pm SD & $2.26 \pm 0.16$ & $2.256 \pm 0.16$ & 0.530 \\
\hline
\end{tabular}

Table (4): ECG rhythm and findings in whole study population

\begin{tabular}{llc}
\hline & & $\mathbf{n}(\%)$ \\
\hline ECG (Rhythm) & Sinus & $90(90.0)$ \\
& Tachyarrhythmia & $10(10.0)$ \\
ECG (finding) & Inverted T wave & $12(12.0)$ \\
& Inverted T wave \& pathological Q & $16(16.0)$ \\
& LVH & $28(28.0)$ \\
& RT VH & $20(20.0)$ \\
& Normal & $2424.0)$ \\
\hline
\end{tabular}


Table (5): Echo parameters pre and post dialysis in patient with intradialytic HTN.

\section{P value}

\begin{tabular}{|c|c|c|c|c|}
\hline \multirow[t]{3}{*}{ LVEDD (mm) } & Pre & Mean \pm SD & $48.3 \pm 4.1$ & $<0.001$ \\
\hline & Post & Mean \pm SD & $46.6 \pm .3$ & \\
\hline & $\%$ change & Median (range) & $-4.2(-10.2-17.2)$ & \\
\hline \multirow[t]{3}{*}{ LVESD (mm) } & Pre & Mean \pm SD & $39 \pm 6.2$ & $<0.001$ \\
\hline & Post & Mean \pm SD & $37.1 \pm 6.1$ & \\
\hline & $\%$ change & Median (range) & $-4.7(-9.0--1.3)$ & \\
\hline \multirow[t]{3}{*}{$\mathbf{E F}$} & Pre & Mean \pm SD & $53.1 \pm 5.2$ & 0.330 \\
\hline & Post & Mean \pm SD & $52.7 \pm 5.3$ & \\
\hline & $\%$ change & Median (range) & $-.7(-15.5-20.7)$ & \\
\hline \multirow[t]{3}{*}{ LAV $\left(\mathbf{m m}^{\mathbf{3}}\right)$} & Pre & Mean \pm SD & $50.6 \pm 9.7$ & $<0.001$ \\
\hline & Post & Mean \pm SD & $40.8 \pm 10.7$ & \\
\hline & $\%$ change & Median (range) & $-21.1(-33.6--4.8)$ & \\
\hline \multirow[t]{3}{*}{$\operatorname{COP}(\mathrm{L} / \mathrm{min})$} & Pre & Mean \pm SD & $5.9 \pm 1.3$ & $<0.001$ \\
\hline & Post & Mean \pm SD & $5.6 \pm 1.3$ & \\
\hline & $\%$ change & Median (range) & $-6.2(-13.9--2.7)$ & \\
\hline \multirow[t]{3}{*}{$\mathbf{S V}(\mathbf{m l})$} & Pre & Mean \pm SD & $82.5 \pm 17.8$ & $<0.001$ \\
\hline & Post & Mean \pm SD & $69.5 \pm 17$ & \\
\hline & $\%$ change & Median (range) & $-14.1(-32.9-5.6)$ & \\
\hline \multirow[t]{3}{*}{ LA Diameter (mm) } & Pre & Mean \pm SD & $38.2 \pm 4.3$ & $<0.001$ \\
\hline & Post & Mean \pm SD & $32.5 \pm 4.2$ & \\
\hline & $\%$ change & Median (range) & $-13.5(-36.4--6.8)$ & \\
\hline
\end{tabular}


Benha medical journal,vol.37, Special issue (Internal medicine and Hepatology),2020

Table (6): Echo parameters pre and post dialysis in patient with intradialytic HTN and non-intradialytic HTN.

\begin{tabular}{|c|c|c|c|c|c|c|c|}
\hline \multirow{2}{*}{$\begin{array}{l}\text { LVEDD } \\
(\mathrm{mm})\end{array}$} & \multicolumn{3}{|c|}{ Intradialytic HTN patient. } & \multicolumn{3}{|c|}{ Non intradialytic HTN patient. } & \multirow{2}{*}{$\begin{array}{l}\text { P value } \\
0.363\end{array}$} \\
\hline & Pre & Mean \pm SD & $48.3 \pm 4.1$ & Pre & Mean \pm SD & $59.64 \pm 2.13$ & \\
\hline & Post & Mean \pm SD & $46.6 \pm .3$ & Post & Mean \pm SD & $58.48 \pm .2 .11$ & 0.046 \\
\hline & $\begin{array}{l}\% \\
\text { change }\end{array}$ & $\begin{array}{l}\text { Median } \\
\text { (range) }\end{array}$ & $-4.2(-10.2-17.2)$ & $\begin{array}{l}\% \\
\text { change }\end{array}$ & $\begin{array}{l}\text { Median } \\
\text { (range) }\end{array}$ & $-1.1(-2-0.7)$ & - \\
\hline \multirow{3}{*}{$\begin{array}{l}\text { LVESD } \\
(\mathbf{m m})\end{array}$} & Pre & Mean \pm SD & $39 \pm 6.2$ & Pre & Mean \pm SD & $39.39 \pm 1.82$ & 0.696 \\
\hline & Post & Mean \pm SD & $37.1 \pm 6.1$ & Post & Mean \pm SD & $39.54 \pm 1.87$ & 0.644 \\
\hline & $\begin{array}{l}\% \\
\text { change }\end{array}$ & $\begin{array}{l}\text { Median } \\
\text { (range) }\end{array}$ & $-4.7(-9.0--1.3)$ & $\begin{array}{l}\% \\
\text { change }\end{array}$ & $\begin{array}{l}\text { Median } \\
\text { (range) }\end{array}$ & $-0.3(-0.2--1.3)$ & - \\
\hline \multirow[t]{3}{*}{$\mathbf{E F}$} & Pre & Mean \pm SD & $53.1 \pm 5.2$ & Pre & Mean \pm SD & $60.55 \pm 2.14$ & 0.272 \\
\hline & Post & Mean \pm SD & $52.7 \pm 5.3$ & Post & Mean \pm SD & $61.05 \pm 2.16$ & 0.188 \\
\hline & $\begin{array}{l}\% \\
\text { change }\end{array}$ & $\begin{array}{l}\text { Median } \\
\text { (range) }\end{array}$ & $-.7(-15.5-20.7)$ & $\begin{array}{l}\% \\
\text { change }\end{array}$ & $\begin{array}{l}\text { Median } \\
\text { (range) }\end{array}$ & $-0.5(0.2+1.4)$ & - \\
\hline \multirow{3}{*}{$\begin{array}{l}\mathbf{L A V} \\
\left(\mathbf{m m}^{3}\right)\end{array}$} & Pre & Mean \pm SD & $50.6 \pm 9.7$ & Pre & Mean \pm SD & $26.740 \pm 1.887$ & 0.054 \\
\hline & Post & Mean \pm SD & $40.8 \pm 10.7$ & Post & Mean \pm SD & $24.80 \pm 1.99$ & 0.026 \\
\hline & $\begin{array}{l}\% \\
\text { change }\end{array}$ & $\begin{array}{l}\text { Median } \\
\text { (range) }\end{array}$ & $-21.1(-33.6--4.8)$ & $\begin{array}{l}\% \\
\text { change }\end{array}$ & $\begin{array}{l}\text { Median } \\
\text { (range) }\end{array}$ & $-1.9(-1.4-2.5)$ & - \\
\hline \multirow{3}{*}{$\begin{array}{l}\text { COP } \\
(\mathrm{L} / \mathrm{min})\end{array}$} & Pre & Mean \pm SD & $5.9 \pm 1.3$ & Pre & Mean \pm SD & $7.67 \pm 0.93$ & 0.931 \\
\hline & Post & Mean \pm SD & $5.6 \pm 1.3$ & Post & Mean \pm SD & $6.80 \pm 0.97$ & 0.873 \\
\hline & $\begin{array}{l}\% \\
\text { change }\end{array}$ & $\begin{array}{l}\text { Median } \\
\text { (range) }\end{array}$ & $-6.2(-13.9--2.7)$ & $\begin{array}{l}\% \\
\text { change }\end{array}$ & $\begin{array}{l}\text { Median } \\
\text { (range) }\end{array}$ & $-0.4(-1.7--0.2)$ & - \\
\hline \multirow[t]{3}{*}{ SV (ml) } & Pre & Mean \pm SD & $82.5 \pm 17.8$ & Pre & Mean \pm SD & $115.4 \pm 7.77$ & 0.341 \\
\hline & Post & Mean \pm SD & $69.5 \pm 17$ & Post & Mean \pm SD & $110.40 \pm 7.80$ & 0.682 \\
\hline & $\begin{array}{l}\% \\
\text { change }\end{array}$ & $\begin{array}{l}\text { Median } \\
\text { (range) }\end{array}$ & $-14.1(-32.9-5.6)$ & $\begin{array}{l}\% \\
\text { change }\end{array}$ & $\begin{array}{l}\text { Median } \\
\text { (range) }\end{array}$ & $-5(-0.5-5.5)$ & - \\
\hline \multirow{3}{*}{$\begin{array}{l}\text { LA } \\
\text { Diameter } \\
(\mathbf{m m})\end{array}$} & Pre & Mean \pm SD & $38.2 \pm 4.3$ & Pre & Mean \pm SD & $45.19 \pm 2.20$ & 0.982 \\
\hline & Post & Mean \pm SD & $32.5 \pm 4.2$ & Post & Mean \pm SD & $42.96 \pm 2.37$ & 0.792 \\
\hline & $\begin{array}{l}\% \\
\text { change }\end{array}$ & $\begin{array}{l}\text { Median } \\
\text { (range) }\end{array}$ & $-13.5(-36.4--6.8)$ & $\begin{array}{l}\% \\
\text { change }\end{array}$ & $\begin{array}{l}\text { Median } \\
\text { (range) }\end{array}$ & $-2.2(-2.8--0.8)$ & - \\
\hline
\end{tabular}





\section{Discussion:}

CKD is a global public health problem with a rising prevalence. Low glomerular filtration rate is associated with higher risk for kidney failure requiring dialysis, as well as with cardiovascular disease (CVD), hypertension, anemia, and other metabolic complications (7).

Echocardiography is an established method for the assessment of left ventricular (LV) and right ventricular function. LV diastolic dysfunction is an important cause of cardiac morbidity in ESRD patients. Diastolic dysfunction appears to be the initial LV dysfunction and might even precede left ventricular hypertrophy (LVH) (8).

$\mathrm{LVH}$ is very frequent in patients with intradialytic hypertension and the left ventricular mass index further progressively increases after the beginning of hemodialysis therapy (9).

Our study showed that there was statistically significant difference in systolic and diastolic blood pressures after hemodialysis as SBP increase in $30 \%$ of patient. SBP increased in from $(128 \pm 10$ to $143 \pm 8)$ and DBP from $(78 \pm 4$ to $87 \pm 4)$ in $30 \%$ of patients.

The prevalence of intradialytic hypertension was provided by an analysis of 438 dialysis patients participating in the Crit-Line Intradialytic Monitoring Benefit (CLIMB) study, in which $13.2 \%$ of participants exhibited intradialytic rise in systolic BP (SBP) >10 mm Hg.( 3)

Patients with intradialytic hypertension tended to be older, received more antihypertensive drugs, had lower dry weight and lower interdialytic weight gain as compared with those without intradialytic hypertension. (3)

In a subsequent analysis from the US Renal Data System Dialysis Morbidity and Mortality Wave II cohort showed that 213 of 1718 patients (12.2\%) experienced intradialytic hypertension, defined as increase in SBP $>10 \mathrm{~mm} \mathrm{Hg}$ from pre to post dialysis. (3)

$\mathrm{Mg}$ is reported to be involved in regulation of blood pressure where $\mathrm{Mg}$ can change vascular tone result in increased blood pressure (10).

$\mathrm{Mg}$ serum is associated with endothelial dysfunction, increased reactivity, increased contractility, vascular remodeling and inflammation, and increased blood pressure (11).

In our study serum $\mathrm{Mg}$ level was $(2.3 \pm 0.2$ $\mathrm{mg} / \mathrm{dL}$ ) with no significant correlation in patient with intradialytic HTN. This is in 
agreement with where serum $\mathrm{Mg}$ level was $(3 \pm 0.5 \mathrm{mg} / \mathrm{dL})$. and (12) where the mean serum magnesium level of $3.61 \mathrm{mg} / \mathrm{dl}$.

In our study serum Ca level was $(7.5 \pm$ $0.5 \mathrm{mg} / \mathrm{dl})$, serum $\mathrm{PO}^{4}$ level was $(5.5 \pm$ $1.5 \mathrm{mg} / \mathrm{dl})$ and serum PTH level was $(363 \pm$ $120 \mathrm{pg} / \mathrm{ml}$ ) with no significant correlation in patient with intradialytic HTN.

As the glomerular filtration rate (GFR) decreases, serum phosphorus increase, followed by a decrease in the serum activated vitamin D level. As a consequence, the serum calcium level decreases.(13)

In our study $\mathrm{HCO} 3$ was $(18 \pm 1 \mathrm{mEq} / \mathrm{Ll})$. This is in agreement with (14) where HCO 3 was $21.9 \mathrm{mEq} / \mathrm{L}$ among 56,385 patients who received hemodialysis from 2001-2003.

Patients on chronic hemodialysis are at greater risk of morbidity and mortality as compared with the general populations.

$\mathrm{LVH}$ on ECG is significant as it is independently associated with adverse cardiovascular outcomes (15).

Our study was done on $100 \mathrm{HD}$ patients showing sinus rhythm in $90 \%$ of patients and sinus tachyarrthmia in $10 \%$ of patients. There was inverted $\mathrm{T}$ wave in $12 \%$ of patients, left ventricular hypertrophy in $28 \%$ of patients, right ventricular hypertrophy in $20 \%$ of patients, normal findings in $24 \%$ of patients. This is in agreement with study done by (16) as LVH was found in $27.6 \%$ of all patients on HD.

Our study showed that there was statistically significant decrease in LVESD from $(39 \pm 6.2 \mathrm{~mm})$ to $(37.1 \pm 6.1 \mathrm{~mm}) \quad(\mathrm{p}$ value $<0.001)$, LVEDD from $(48.3 \pm 4.1$ $\mathrm{mm})$ to $(46.6 \pm 3 \mathrm{~mm})(\mathrm{p}$ value $<0.001)$, LA diameter from $(38.2 \pm 4.3 \mathrm{~mm})$ to $(32.5 \pm 4.2 \mathrm{~mm}) \quad(\mathrm{p}$ value $<0.001)$ in patient with IDH .This is in agreement with (17) where they studied 84 patients on regular hemodialysis (HD) with IDH (mean age of $45.3 \pm 14.5$ years). Standard echocardiographic measurements were obtained and Left ventricle and left atrium diameter were significantly decreased after HD. Also, (18) study demonstrated that Left ventricular end-diastolic dimension (LVEDD) decreased from $40.3 \pm 4.2 \mathrm{~mm}$ (mean \pm standard deviation) $\mathrm{mm}$ to $36.1 \pm$ $4.6 \mathrm{~mm}$ in patient with IDH. Our study showed that there was statistically significant decrease in COP from $(5.9 \pm$ $1.3 \mathrm{~L} / \mathrm{m})$ to $(5.6 \pm 1.3 \mathrm{~L} / \mathrm{m}) \quad(\mathrm{p}$ value $<$ $0.001), \mathrm{SV}$ from $(82.5 \pm 17.8 \mathrm{ml})$ to $(69.5$ $\pm 17 \mathrm{ml})(\mathrm{p}$ value $<0.001)$, LA volum from $(50.6 \pm 9.7 \mathrm{~mm} 3)$ to $(40.8 \pm 10.7$ $\mathrm{mm} 3) \quad(\mathrm{p}$ value $<0.001)$ in patient with IDH .This is in agreement with (19) as 
COP decrease from $(6.47 \pm 0.9 \mathrm{~L} / \mathrm{m})$ to $(5.47 \pm 0.8 \mathrm{~L} / \mathrm{m}) \quad(\mathrm{p}$ value $<0.001)$ In Ercan etal ., (2016) LA volum decrease from $(36.7 \pm 21.7 \mathrm{~mm} 3)$ to $(29.5 \pm 10.0 \mathrm{~mm} 3) \quad(\mathrm{p}$ value $<0.001$ ) and this agreement with our study . In (20) LA volum decrease from $(36.7 \pm 21.7 \mathrm{~mm} 3)$ to $(29.5 \pm 10.0 \mathrm{~mm} 3) \quad(\mathrm{p}$ value $<0.001)$ and this agreement with our study . In (21)SV decrease from (57.3 \pm $30.1 \mathrm{ml})$ to $(56 \pm 29.1 \mathrm{ml})$ and this agree with our study. In non-intradialytic HTN Our study showed that there was statistically significant decrease in LVESD from $(39.39 \pm 1.82 \mathrm{~mm})$ to $(39.54$ $\pm 1.87 \mathrm{~mm}) \quad(\mathrm{p}$ value $<0.011), \quad$ LVEDD from $(59.64 \pm 2.13 \mathrm{~mm})$ to $(58.48 \pm .2 .11$ $\mathrm{mm})$ ( $\mathrm{p}$ value <0.001) , SV from (115.4 $\pm 7.77 \mathrm{ml})$ to $(110.40 \pm 7.80 \mathrm{ml})$ (p value $<0.001)$, LA diameter from $(45.19$ $\pm 2.20 \mathrm{~mm})$ to $(42.96 \pm 2.37 \mathrm{~mm}) \quad$ (p value $<$ $<0.001)$, , LA volume from $(26.740$ $\pm 1.887 \mathrm{~mm} 3)$ to $(24.80 \pm 1.99 \mathrm{~mm} 3)$ (p value $<0.001$ ) in non-intradialytic HTN patient. This is in agreement with (22) where they studied 166 patients on regular hemodialysis, and found a decrease in LVESD from $(36.7 \pm 7.2 \mathrm{~mm})$ to $(36.4$ $\pm 6.4 \mathrm{~mm}$ ) ( $\mathrm{p}$ value $<0.011$ ). LVEDD from $(56.6 \pm 6.5 \mathrm{~mm})$ to $(54.8 \pm 6.5 \mathrm{~mm})$ (p value $<0.001)$, SV from $(99.8 \pm 22.9 \mathrm{ml})$ to $(93.7 \pm$ $27.8 \mathrm{ml})$ ( $\mathrm{p}$ value $<0.001)$.
Our results agree with the findings of (23) and (24) who found that LV end-diastolic and end systolic size significantly decreased along with a decrease in volume after HD. This is also in agreement with (25) study where there was a decrease in LVESD from $(48.7 \pm 28.1 \mathrm{ml})$ to $(38.7 \pm$ 24ml) (p value <0.011), LVEDD from $(132.7 \pm 47.2 \mathrm{ml})$ to $(112.6 \pm 40.1 \mathrm{ml})(\mathrm{p}$ value $<0.001)$, LA diameter from (41.8 \pm $7.5 \mathrm{~mm})$ to $(39.3 \pm 8 \mathrm{~mm}) \quad$ (p value $<$ $<0.001)$, LA volume from $(23.8 \pm 6.95$ $\mathrm{mm} 3)$ to $(21.7 \pm 6.7 \mathrm{~mm} 3) \quad$ (p value $<0.001$ ) in non-intradialytic HTN patient .

\section{Conclusion}

IDH is a common (prevalence 30\%) alteration in the dialysis population. This alteration predicts a high death risk. Volume and sodium overload, endothelial dysfunction, overactivity of the RAS and SNS all play a role in this alteration. There was significant decrease in LVEDD, LVESD, LAV, COP, SV and LA diameter in HD patient with IDH.

\section{References}

1. Flythe J. E., Inrig J. K., Shafit., Chang T. I., Cape K., Dinesh, et al . (2013). Association of intradialytic blood pressure variability with increased all-cause and cardiovascular mortality in patients treated with long-term hemodialysis. 
American Journal of Kidney Diseases, 61(6),966974

2. Daugirdas J. T., Black P.G., Ing T.S. (2007) In "Handbook of Dialysis". 4th ed. Philadelphia, PA:Lippincott Williams \& Wilkins, a Wolter Kluwer Business;.

3. Park J., Rhee C. M., Sim J. J., Kim Y. L., Ricks J., Streja, et al (2013). A comparative effectiveness research study of the change in blood pressure during hemodialysis treatment and survival. Kidney international, 84(4), 795-802.

4. Inrig JK, Patel UD, Toto RD and Szczech LA. Association of blood pressure increases during hemodialysis with 2-year mortality in incident hemodialysis patients: a secondary analysis of the Dialysis Morbidity and Mortality Wave 2 Study. Am J Kidney Dis. 2009;54:881-890. doi: 10.1053/j.ajkd.2009.05.012.

5. Davenport, A. Response to "The long forgotten salt factor”. Kidney Int. 74, 964-965 (2008).

6. Krapf, R. \& Hulter, H. N. Arterial hypertension induced by erythropoietin and erythropoiesisstimulating agents (EsA). Clin. J. Am. Soc. Nephrol. 4, 470-480 (2009).

7. Muntner P, Judd SE, Gao L, Gutiérrez OM, Rizk DV, McClellan W, et al (2013). Cardiovascular risk factors in CKD associate with both ESRD and mortality. J Am Soc Nephrol; 24:1159-1165.

8. Agarwal R (2013) B-type natriuretic peptide is not a volume marker among patients on hemodialysis. Nephrol Dial Transplant 28(12):3082-3089

9. Foley R. N., Curtis B. M., Randell E. W., \& Parfrey P. S. (2010). Left ventricular hypertrophy in new hemodialysis patients without symptomatic cardiac disease. Clinical Journal of the American Society of Nephrology, 5(5), 805-813.
10. Grober U, Schmidt J and Kisters K (2015). Magnesium in prevention and therapy. Nutrients;7:8199-8226.

11. Pakfetrat M, Roozbeh J, Malekmakan N, Nasab $\mathrm{M}$ and Nikoo M.. Is there an association between intradialytic hypotension and serum magnesium changes?. Hemodialysis International 2010;14:492497.

12. Amit Naik, Rakesh.S. Jagat, P and Dhurvey. Study of serum magnesium level in Acute kidney injury and Chronic kidney disease and its clinical correlation with other biochemical parameters. Journal of Evolution of Medical and Dental Sciences. 2015; 4: 12928-32.

13. Group KDIGOC-MUW. KDIGO (2017) clinical practice guidelines update for the diagnosis, evaluation, prevention, and treatment of chronic kidney disease-mineral and bone disorder (CKDMBD). Kidney Int Suppl 2017; 7:1-59.

14. Wu DY, Shinaberger CS, Regidor DL, McAllister CJ, Kopple JD and Kalantar-Zadeh K (2006).: Association between serum bicarbonate and death in hemodialysis patients: Is it better to be acidotic or alkalotic? Clin J Am Soc Nephrol 1: 70-78

15. Weiner DE, Tighiouart H, Vlagopoulos PT, Griffith JL, Salem DN, Levey AS, et al. Effects of anemia and left ventricular hypertrophy on cardiovascular disease in patients with chronic kidney disease. J Am Soc Nephrol 2005;16(6):1803-10.

16. Chijioke A, Makusidi AM and Kolo PMb (2012). Electrocardiographic abnormalities among dialysis naïve chronic kidney disease patients in Ilorin Nigeria. Ann Afr Med;11(1):21-6.

17. Gerede D. M., Turhan S., Kaya C. T., Ozcan O. U., Goksuluk H., VurgunV. K., etal ., (2015). 
Effects of hemodialysis on Tei Index: comparison between flow Doppler and tissue Doppler imaging. Echocardiography, 32(10), 1520-1526.

18. Kudoh A and Yasuo M .(2014).The dual effects of hemodialysis on cardiac function assessed by pulsed Doppler echocardiography. Japanese circulation journal ; 52(1) : 13-20.

19. Krupička J., Janota T., Kasalova Z., \& Hradec, J. (2009). Natriuretic peptides-physiology, pathophysiology and clinical use in heart failure. Physiological research, 58(2).

20. Ene-Iordache, B., Perico, N., Bikbov, B., Carminati, S., Remuzzi, A., Perna, etal. (2016). Chronic kidney disease and cardiovascular risk in six regions of the world (ISN-KDDC): a crosssectional study. The Lancet Global Health, 4(5), e307-e319.

21. Michael J. Germain , Jyovani JOUBERT, Daniel O'GRADY, Brian H. NATHANSON, Yossi CHAIT, Nathan W LEVIN,(2018) Original Article Cardiovascular Comparison of stroke volume measurements during haemodialysis using bioimpedance cardiograph and echocardiography Haemodialysis International2018;22:201-208
22. London, G. M., Pannier, B., Guerin, A. P., Blacher, J., Marchais, S. J., Darne, B.,etal. (2001). Alterations of left ventricular hypertrophy in and survival of patients receiving hemodialysis: followup of an interventional study. Journal of the American Society of Nephrology, 12(12), 27592767.

23. Galetta F, Cupisti A, Franzoni F, Femia FR, Rossi M, Bar-sotti G etal 2005: Left ventricular function and calciumphosphate plasma levels in uraemic patients.J Intern Med258: 378-384, 2005

24. Hung, K. C., Huang, H. L., Chu, C. M., Chen, C. C., Hsieh, I. C., Chang, S. T.,etal . (2004). Evaluating preload dependence of a novel Doppler application in assessment of left ventricular diastolic function during hemodialysis. American journal of kidney diseases, 43(6), 1040-1046.

25. Ersbøll, M., Raja A. A., Warming P. E., Nielsen, T. L., Plesner, L. L., Dalsgaard, M., etal. (2019). Changes in left ventricular filling parameters before and after dialysis in patients with end stage renal disease. The international journal of cardiovascular imaging, 1-9.

To cite this article: Mohamed E. Ibrahim, EL-Metwally L. EL-Shehawy, Hassan G. Abdelsalam, Ahmed E. Mansour, Nashwa G. Mohamed. Myocardial functional disorders in hemodialysis patient with intradialytic hypertension. BMFJ 2020; 37 (internal medicine and Hepatology): 24-36. DOI: 10.21608/bmfj.2020.18583.1128 
Original article

DOI: 10.21608/bmfj.2020.18583.1128 